\title{
E-Commerce Development Risk Evaluation Using MCDM Techniques
}

\author{
Salem Alharbi, Mohsen Naderpour \\ Global Big Data Technologies Centre \\ Faculty of Engineering and Information Technology \\ University of Technology Sydney \\ P.O. Box 123, Broadway, NSW 2007, Australia \\ Salem.Alharbi@student.uts.edu.au, Mohsen.Naderpour@uts.edu.au
}

\begin{abstract}
Electronic commerce (EC) development takes place in a complex and dynamic environment that includes high levels of risk and uncertainty. This paper proposes a new method to assess the risks associated with EC development using multi-criteria decision-making techniques A model based on analytic hierarchy process (AHP) and the technique for order of preference by similarity to ideal solution (TOPSIS) is proposed to assist EC project managers and decision makers in formalizing the types of thinking that are required in assessing the current risk environment of their EC development in a more systematic manner than before. The solution includes the use of AHP for analyzing the problem structure and determining the weights of risk factors. While the TOPSIS technique helps to obtain a final ranking among projects. The results of an evaluation show the usefulness performance of the method.
\end{abstract}

Keywords: E-Commerce, Risk Analysis, Multi-Criteria Decision Making, AHP, TOPSIS.

\section{Introduction}

Electronic commerce (EC) may be the most promising information technology application to emerge in recent years. EC addresses the needs of organizations, suppliers and customers to reduce costs while improving the quality of goods and services and increasing the speed of service delivery [1]. The current highly competitive business environment demands a good quality EC system; however, EC development is subject to various types of risk. Indeed, a task that is critical to the proper management of EC development is the assessment of risk. An important step in advancing our knowledge requires that we understand and address these risks.

The concept of risk became popular in economics during the 1920s. Since then, it has been successfully used in theories of decision making in economics, finance, and the decision science. The risk is defined as the "possibility of loss or injury" or "someone or something that creates or suggests a hazard". At present, there is no agreed upon universal definition of EC risk but information security is a widely recognized aspect of EC risk [2]. 
Before conducting risk analysis, risk factors associated with EC development must be identified. Several empirical researches such as [3] and [5] have focused on identifying the potential risk factors that threaten EC development. In the study of Wat et al. [3], a source-based approach to categorizing EC development risks is initially used, with technical, organizational, and environmental risks as three primary source categories. Then the potential risks associated with EC development was identified with 51 risk items based on a comprehensive literature review and interviews with EC practitioners. After an empirical study, 10 major dimensions of risks associated with EC development, namely: (1) resources risk, (2) requirements risk, (3) vendor quality risk, (4) client-server security risk, (5) legal risk, (6) managerial risk, (7) outsourcing risk, (8) physical security risk, (9) cultural risk, and (10) re-engineering risk. Ngai and Wat [2] have used this classification and developed a web-based fuzzy decision support system for risk assessment. Leung et al., [4] have developed an integrated knowledge-based system that assists project managers to determine potential risk factors. In another study, Addison [5] used a Delphi technique to collect the opinion of experts and proposed 28 risks for EC projects. Meanwhile, Carney et al. [6] have identified four categories comprising 21 risk areas, and designed a risk evaluation tool in this matter. Cortellessa et al., [7] have introduced a methodology which elaborates annotated UML diagrams to estimate the performance failure probability, and combines it with the failure severity estimate which is obtained using the functional failure Analysis. This methodology is still have some limitation and only suitable for the analysis of performance-based risk in the early phases of the software life cycle.

This paper develops a new risk evaluation method that can be used to effectively support EC project managers in conducting risk assessment in EC development. The idea relies upon the use of analytic hierarchy process (AHP) and the technique for order of preference by similarity to ideal solution (TOPSIS), two popular multicriteria decision making (MCDM) techniques, in an innovative manner.

The paper is organized as follows. The background to this study is presented in Section 2. Section 3 shows our EC development risk evaluation method. The performance of the proposed method is illustrated in Section 4. Sections 5 and 6 summarize the discussion and conclusion.

\section{Background}

\subsection{E-Commerce}

Over the last two decades, the popularity of the Internet and network technology has increased rapidly. Consequently, EC has become a common activity in modern business operations [8]. The growth of EC activities in the last 20 years has attracted attention from academics as well as practice in various fields. For example, computer science researchers have shown interest in the technical and system sides of EC. Law academics are interested in legal issues relative to EC. Business research focuses on marketing and management issues of EC while social research focuses on the 
influence of EC on human beings and society. From different perspectives, EC is differently referred to [1]:

- Communication perspective: EC is the delivery of services/products, payments or information via telephone lines, computer networks or other means.

- Business process perspective: $\mathrm{EC}$ is the technology application for automating business workflows and transactions.

- Service perspective: EC is a tool to address the desire of consumers, management and firms to reduce service costs while increasing quality of goods and service delivery speed.

- Online perspective: EC helps to sell and buy information and products on the Internet and with online services.

\subsection{Multi-Criteria Decision-Making Techniques}

Description of MCDM problems. MCDM is concerned with structuring and solving decision and planning problems involving multiple criteria. There are some common characteristics in MCDM problems, such as the presence of multiple noncommensurable and conflicting criteria, different units of measurement among the criteria, and the presence of disparate alternatives. All of the criteria in an MCDM problem can be classified into two categories. Criteria that are to be maximized are benefit criteria. In contrast, criteria that are to be minimized fall into the cost criteria category. A typical MCDM problem based on $m$ alternatives $\left(A_{1}, A_{2}, \ldots, A_{m}\right)$ and $n$ criteria $\left(C_{1}, C_{2}, \ldots, C_{n}\right)$ can be presented as follows [9]:

$$
\boldsymbol{X}=\left[x_{i j}\right]_{\mathrm{m} * \mathrm{n}}, \boldsymbol{W}=\left[w_{j}\right]_{\mathrm{n}}
$$

where $X$ is the decision matrix, $x_{i j}$ is the performance of the $i$ th alternative with respect to the $j$ th criterion, $\boldsymbol{W}$ is the weight vector, and $w_{j}$ is the weight of the $j$ th criterion. The original decision matrix $\boldsymbol{X}$ is typically incomparable because different criteria are expressed using different units of measure. Therefore, data should be transformed into comparable values using a normalization procedure. The weight vector $\boldsymbol{W}$ has a large effect on the ranking results of alternatives. It is usually fixed using an expert's assignment or the AHP method [9].

AHP. AHP is a structured technique for organizing and analyzing complex decisions, based on mathematics and psychology. It was developed by Thomas L. Saaty in the 1970s and has been extensively studied and refined since then. AHP relies on the judgment of an expert to establish a priority scale and uses pairwise comparisons. It is a highly popular MCDM method, and it has certain advantages and disadvantages [10]. One of the biggest advantages of AHP is that it is easy to use. As this method utilizes pairwise comparisons, it allows the coefficients to be weighted and the alternatives to be easily compared. Due to its hierarchical structure, it is scalable and can be adjusted in size to accommodate decision-making problems. AHP requires data for pairwise comparison, but it is not as data intensive as other MCDM methods such as multi-attribute utility theory. One of the common limitations of AHP is that it experiences problems related to the interdependence between the criteria and alternatives [11]. The AHP approach involves pairwise comparison, and thus it is 
vulnerable to inconsistencies in ranking criteria and judgment. AHP evaluates each instrument in comparison with the other instruments and does not grade any instrument in isolation. Therefore, the approach is unable to properly identify weaknesses and strengths associated with each instrument [12]. AHP is susceptible to rank reversal in its general form, and this is another major drawback. As rankings are used for comparison, addition of alternatives at the finish of the process can result in the final rankings to reverse or flip. AHP has been used extensively for resource management, performance-type problems, corporate strategy and policy, political strategy, public policy and planning. Problems related to resource management have a limited number of alternatives, which minimizes the disadvantage of rank reversal.

AHP breaks the decision-making process into steps so that decisions can be made in an organized way with defined priorities. The steps for decision-making in AHP are as follows [10]:

Step 1. Define the problem: In this step, the problem is defined and the goal is determined.

Step 2. Represent the problem graphically: Information is arranged into a hierarchical structure. The decision goal is stated, the criteria are defined and the alternatives are identified.

Step 3. Develop a judgment preference: This is done to measure the preference for alternatives against the criteria. A pairwise comparison is made on a scale of one to nine to rate the relative preference.

Step 4. Relative weight calculation: The relative weights of the alternatives and criteria are determined through calculations. The eigenvalue technique is used to calculate the priority vector or relative weight, where $\mathrm{P}$ is the priority vector.

Step 5. Synthesis: By aggregating the weights in the results vertically, the contribution of every alternative to the overall goal is computed. An overall ranking of the alternatives is obtained through combining priority vectors of all the criteria.

Step 6. Consistency: The consistency ratio is used to measure the accuracy of the decision.

TOPSIS. Among various methods that have been established to solve real-world MCDM problems, TOPSIS works satisfactorily in various situations and can be applied to diverse areas. Hwang and Yoon were the first to propose this technique, which can be used for selecting and evaluating the best alternative for a problem [13]. It has became a well-known classical MCDM method, and has gained ample interest from practitioners and researchers around the world.

TOPSIS is a ranking method that is easy to understand and apply. It aims to select alternatives that are farthest from the negative ideal solution and closest to the positive ideal solution. The advantage of choosing the positive ideal solution is that it minimizes the cost criteria and maximizes the benefit criteria, whereas the negative ideal solution minimizes the benefit criteria and maximizes the cost criteria. The use of TOPSIS helps in arranging alternatives cardinally, making maximum use of attribute information; therefore, it does not require independent attribute preference [14]. The application of TOPSIS requires attribute values to be monotonically decreasing or increasing, numeric and to have commensurable units. Fig. 1 shows the procedure for implementing TOPSIS [15]. First, the initial decision matrix is formed, and then the decision matrix is normalized. In the second step, the weighted 
normalized decision matrix is constructed, and this matrix is then used in the third step to determine the negative and positive ideal solutions. In the fourth step, the measures of separation for every alternative are calculated. In the fifth and final step, the coefficient of relative closeness is computed. Then, according to the value of the coefficient of closeness, alternatives are ranked or arranged in increasing order. The TOPSIS process is performed as follows [9]:

Step 1. Normalize the decision matrix:

$$
r_{i j}=\frac{x_{i j}}{\sqrt{\sum_{i=1}^{m} x_{i j}^{2}}}, i=1, \ldots, m ; j=1, \ldots, n
$$

where $r_{i j}$ denotes the normalized value of the $j$ th criterion for the $i$ th alternative $A_{i}$.

Step 2. Calculate the weighted normalized decision matrix:

$$
v_{i j}=r_{i j} * w_{j}, i=1, \ldots, m ; j=1, \ldots, n
$$

where $w_{j}$ is the weight of the $j$ th criterion or attribute.

Step 3. Determine the positive ideal and negative ideal solutions:

$$
\begin{aligned}
& A^{+}=\left(v_{1}^{+}, \ldots, v_{n}^{+}\right) \\
& A^{-}=\left(v_{1}^{-}, \ldots, v_{n}^{-}\right)
\end{aligned}
$$

where $A^{+}$denotes the positive ideal solution, and $A^{-}$denotes the negative ideal solution. If the $j$ th criterion is a beneficial criterion, then $v_{j}^{+}=\max \left\{v_{i j}\right.$, $i=1, \ldots, m\}$ and $v_{j}^{-}=\min \left\{v_{i j}, i=1, \ldots, m\right\}$. In contrast, if the $j$ th criterion is a cost criterion, then $v_{j}^{+}=\min \left\{v_{i j}, i=1, \ldots, m\right\}$ and $v_{j}^{-}=\max \left\{v_{i j}, i=1, \ldots, m\right\}$.

Step 4. Calculate the distances from each alternative to a positive ideal solution and a negative ideal solution:

$$
\begin{aligned}
& D_{i}^{+}=\sqrt{\sum_{j=1}^{n}\left(v_{i j}-v_{j}^{+}\right)^{2}}, i=1, \ldots, m \\
& D_{i}^{-}=\sqrt{\sum_{j=1}^{n}\left(v_{i j}-v_{j}^{-}\right)^{2}}, i=1, \ldots, m
\end{aligned}
$$

where $D_{i}^{+}$denotes the distance between the $i$ th alternative and the positive ideal solution, and $D_{i}^{-}$denotes the distance between the $i$ th alternative and the negative ideal solution.

Step 5. Calculate relative closeness to the ideal solution. 


$$
C_{i}=\frac{D_{i}^{-}}{D_{i}^{+}+D_{i}^{-}}, i=1, \ldots, m
$$

Step 6. Rank the alternatives, sorting by the value $C_{i}$ in decreasing order.

\section{The E-Commerce Development Risk Evaluation Method}

As discussed earlier, EC offers many opportunities for business, and EC projects have many advantages. Even so, EC development is subject to various risks, and it is essential to manage these risks to avoid problems. The assessment of risk is thus essential to the proper management of EC projects. This section develops the risk evaluation method for EC development. Firstly, the risk factors are introduced in Section 3.1. Secondly, the method is presented in Section 3.2.

\subsection{Risk Factors}

Several empirical researches have been carried out examining the risk factors that threaten EC project development. More than 50 risk factors have been identified in the literature. However, considering all of them is not practical in any situations. In this paper, 12 important risk factors based on our expert's knowledge, who has seven years of professional experience in EC projects management, have been considered. The factors are categorized in three groups as shown in Fig. 1 including: Technical, organizational and environmental risks. In addition, 12 major risk factors are determined as shown in Table 1. The table shows the risk categories and potential risks associated with EC development in each category.

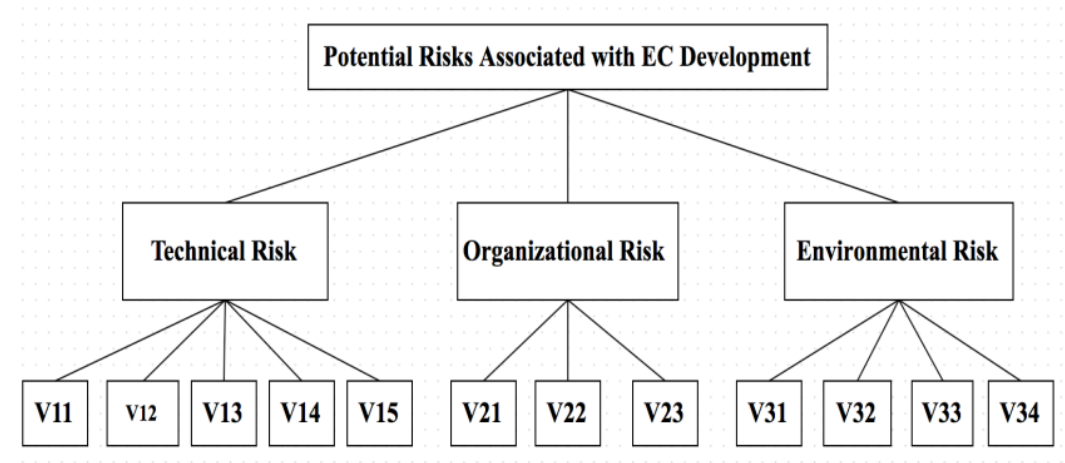

Fig 1. The hierarchy structure of EC development risks. 
Table 1. Potential risks associated with EC development.

\begin{tabular}{lcl}
\hline Category & Variable & Potential Risk \\
\hline \multirow{3}{*}{ Technical Risk } & V11 & Project complexity \\
& V12 & Software or hardware problem-caused system failure \\
& V13 & Poor design, code or maintenance procedure \\
& V14 & Wrong functions and properties development \\
& V15 & Continuous change of system requirements \\
\hline \multirow{3}{*}{ Organizational Risk } & V21 & Wrong schedule estimation \\
& V22 & Project over budget \\
& V23 & Lack of expertise and experience in EC \\
\hline \multirow{4}{*}{ Environmental Risk } & V31 & Lack of international legal standards \\
& V32 & Difficult to change outsourcing decision/vendor \\
& V33 & Loss of data control \\
& V34 & Different users with different cultures \\
\hline
\end{tabular}

\subsection{Method}

The risk evaluation method as shown in Fig. 2 consists of two parts: (1) determining the weights of the criteria, and (2) evaluating the alternatives. The goal is to select the project that has the least amount of associated risk. The risk factors identified in previous section are considered as criteria. Therefore, the pairwise comparison of AHP is used to calculate the weights of the criteria and sub-criteria. Once the weights have been obtained, the evaluation of the alternatives is carried out using TOPSIS methodology.

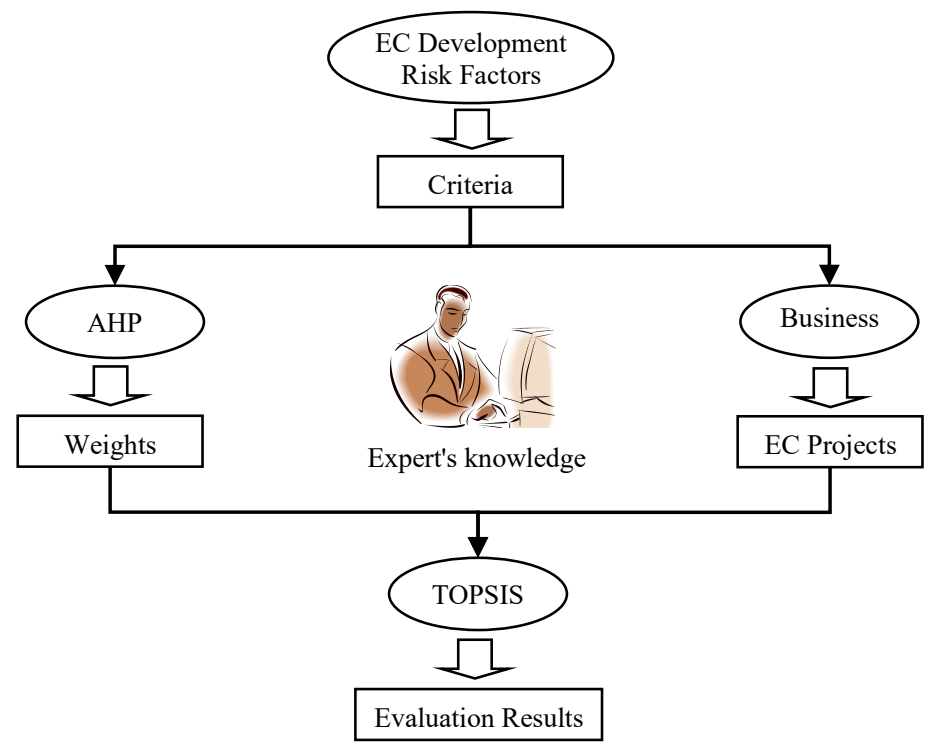

Fig 2. The EC risk evaluation method. In order to determine the weights of the risk factors, the AHP methodology is applied. Once the weights have been obtained, the evaluation of the EC projects can be carried out using the TOPSIS methodology. 


\section{Application}

EC projects generally have a high IT content (around 20-40\%), but they differ from IT projects in various ways. They are relatively low in cost and are characterized by high business impact. Moreover, EC projects have complex architecture and a high need for fault tolerance and scalability. They require a high rate of continuous evolution, which is driven by competitive actions, technology changes and business model innovations. EC projects are widely used to promote retailing. In the following, three EC projects of a private company are introduced. The projects include an online shopping mall, an online auction, and an online bookstore.

\subsection{Projects of Interest}

- Online shopping mall: The company would like to develop an online multivendor shopping mall that will allow customers to purchase products from participating online stores. The online mall will allow prospective customers to select products from a range of categories/subcategories and given them the opportunity to compare products from various vendors and easily view special offers before adding their choices to a shopping cart and participating in a secure transaction. Prospective vendors should be able to easily pay an annual registration fee, create their own online stores and take control of their stores, add process orders and products from customers.

- Online auction: The online auction is a group that would be established for the purpose of auctions. If an individual wants to sell something via an auction, they would post on that website. The business is simply a selling of products, which gives the project user ability to bid on particular services or products. The primary objective of the e-auction process will be to obtain the highest price and best value. It is not possible to achieve the best value outcomes when the focus remains on price. There are two categories of persons in this project: customers and vendors. Both have their own registration forms. Vendors can sell their products on this website, and customers will purchase them. Each product will be awarded to the customer who places the highest bid on the product.

- Online Bookstore: This project's main objective is to create an online bookstore. This bookstore will give users a chance to search and purchase books online on author, title and subject. Tabular format is used to display selected books, and online bookstore will facilitate the user to make online purchases through credit card. Customer using this website can easily purchase book online and don't need to visit a bookstore and waste time.

\subsection{Weighting Criteria Using AHP}

Based on the proposed method, the ratings are obtained from an expert who has seven years of professional experience in EC project management and is familiar with legal 
services. The matrices are formed and priorities are synthesized using AHP methodology. Table 2 shows the expert' knowledge in pairwise comparison of categories. Then, the weight for the risk categories are calculated by AHP.

Table 2. Pairwise comparison matrix for risk categories.

\begin{tabular}{lccccc}
\hline & Technical Risk & $\begin{array}{c}\text { Organizational } \\
\text { Risk }\end{array}$ & $\begin{array}{c}\text { Environmental } \\
\text { Risk }\end{array}$ & $\begin{array}{c}\text { Geometric } \\
\text { Mean }\end{array}$ & Normalized \\
\hline Technical Risk & 1.00 & 0.25 & 0.17 & 0.347 & 0.089 \\
$\begin{array}{c}\text { Organizational } \\
\text { Risk }\end{array}$ & 4.00 & 1.00 & 0.50 & 1.260 & 0.323 \\
$\begin{array}{c}\text { Environmental } \\
\text { Risk }\end{array}$ & 6.00 & 2.00 & 1.00 & 2.289 & 0.588 \\
& & & SUM & 3.896 & 1.000 \\
\hline
\end{tabular}

The results in Table 2 have been summarized in Fig. 3. As can be seen, the environmental risk is the most important risk category. It is followed by organizational risk and technical risk.

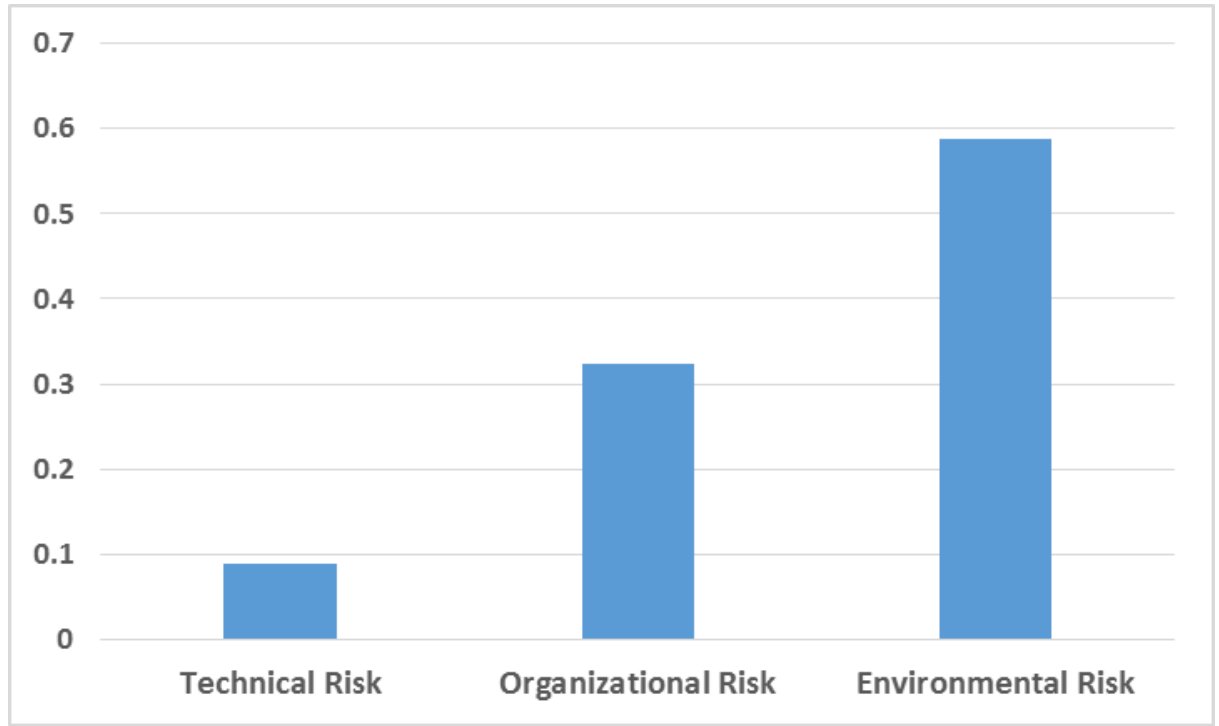

Fig 3. The calculated weights for each risk category.

The complete priority weighting and ranking of the risks are shown in Table 3. The results show that lack of international legal standards is the most significant risk (0.305), and project complexity is the least significant risk. Overall environmental risks have the highest global priority weighting as mentioned before. 
Table 3. The weight of each sub-criteria.

\begin{tabular}{lc}
\hline Sub-category & Technical Risk \\
\hline Project complexity & 0.003 \\
Software or hardware problem-caused system failure & 0.011 \\
Poor design, code or maintenance procedure & 0.024 \\
Wrong functions and properties development & 0.006 \\
Continuous change of system requirements & 0.045 \\
\hline Sub-category & Organizational Risk \\
\hline Wrong schedule estimation & 0.030 \\
Project over budget & 0.070 \\
Lack of expertise and experience in EC & 0.223 \\
\hline Sub-category & Environmental Risk \\
\hline Lack of international legal standards & 0.305 \\
Difficult to change outsourcing decision/vendor & 0.173 \\
Loss of data control & 0.072 \\
Different users with different cultures & 0.038 \\
\hline & SUM \\
\hline
\end{tabular}

\subsection{Ranking EC Projects Using TOPSIS}

Table 4 shows the matrix based on the expert's score for each EC project in each risk factor. We start by calculating the weighted normalized matrix to find the ideal solutions.

Table 4. The expert's opinion for EC projects under each risk factor.

\begin{tabular}{lccccc}
\hline Technical Risk & & & & & \\
\hline Sub-category & V11 & V12 & V13 & V14 & V15 \\
Global weight (criteria*sub-criteria) & 0.003 & 0.011 & 0.024 & 0.006 & 0.045 \\
Project 1: Online shopping mall & 1 & 5 & 6 & 5 & 7 \\
Project 2: Online auction & 4 & 3 & 5 & 3 & 1 \\
Project 3: Online bookstore & 2 & 5 & 2 & & 1 \\
\hline Organizational Risk & & & & V23 \\
\hline Sub-category & V21 & & 0.07 & 0.223 \\
Global weight (criteria*sub-criteria) & 0.03 & 6 & 6 \\
Project 1: Online shopping mall & 4 & 6 & 2 & 6
\end{tabular}




\begin{tabular}{lcccc} 
Project 3: Online bookstore & 3 & 1 & 7 \\
\hline Environmental Risk & & & & V32 \\
\hline Sub-category & V31 & V32 33 & 0.038 \\
Global weight (criteria*sub-criteria) & 0.305 & 0.173 & 0.072 & 4 \\
Project 1: Online shopping mall & 1 & 5 & 1 & 5 \\
Project 2: Online auction & 7 & 1 & 1 & 5 \\
Project 3: Online bookstore & 6 & 3 & 2 & 5 \\
\hline
\end{tabular}

Several matrices including square roots of all sub-criteria, the weighted normalized, distance to positive ideal solution, and distance to negative ideal solution are omitted for space consideration. Table 5 shows the relative closeness to the ideal solution for each project. As can be seen, the online shopping mall is considered as the best solution for this case.

Table 5. The relative closeness to the ideal solution.

\begin{tabular}{lc}
\hline Alternative & TOPSIS Scores \\
\hline Project 1: Online shopping mall & 0.599705 \\
Project 2: Online auction & 0.397074 \\
Project 3: Online bookstore & 0.345942 \\
\hline
\end{tabular}

\section{Discussion}

In this study, several important risk factors threaten EC projects have been identified. In the proposed method, AHP was used for finding the priority weight of the risks involved, but it was not used for ranking the alternatives because it would require a large number of matrices. Instead, TOPSIS, which avoids the complexity related to AHP, was used for ranking the alternative EC projects. TOPSIS is also simpler and faster than AHP in application and makes it possible to treat the qualitative variables quantitatively. This helps the decision-making become simpler by providing positive and negative ideal solutions, allowing the decision-maker to easily determine which alternative to choose. Moreover, TOPSIS is not limited to the number of criteria that are being applied. A case study containing three EC projects for a particular company have been considered for risk evaluation.

\section{Conclusion and Future Work}

The paper developed an approach for selecting EC projects with the minimum risks. AHP methodology was used to calculate the weights of the different risk factors 
contributing to EC projects. TOPSIS methodology was applied to rank the alternatives, thus allowing selection of the best alternative project. The results show the significance of AHP and TOPSIS in solving MCDM problems in EC projects when managers are concerned about choosing the alternative with the lowest risks and maximum benefits.

There are various future directions for this research. Fuzzy logic can be used for assessing risks of EC projects. Fuzzy logic can allow mangers to use linguistic variables and this can help them to do better risk assessment such as conducting FMEA analysis. Fuzzy logic can be used along with AHP and TOPSIS to solve complex risk problems in EC projects. We will also build a prototype based on the proposed method. The results will be compared with other EC risk assessment methodologies as well.

\section{References}

1. Kalakota, R., Whinston, A.B.: Electronic commerce: a manager's guide. Addison-Wesley Professional (1997)

2. Ngai, E.W.T., Wat, F.K.T.: Fuzzy decision support system for risk analysis in ecommerce development. Decision Support Systems 40, 235-255 (2005)

3. Wat, F., Ngai, E.W., Cheng, T.E.: Potential risks to e-commerce development using exploratory factor analysis. International Journal of Services Technology and Management 6, 55-71 (2005)

4. $\quad$ Leung, H.M., Rao Tummala, V.M., Chuah, K.B.: A knowledge-based system for identifying potential project risks. Omega 26, 623-638 (1998)

5. Addison, T.: E-commerce project development risks: Evidence from a Delphi survey. International Journal of Information Management 23, 25-40 (2003)

6. Carney, D.J., Morris, E.J., Place, P.R.: Identifying commercial off-the-shelf (COTS) product risks: the COTS usage risk evaluation. Carnegie-Mellon Univ Pittsburgh (2003)

7. Cortellessa, V., Goseva-Popstojanova, K., Appukkutty, K., Guedem, A.R., Hassan, A., Elnaggar, R., Abdelmoez, W., Ammar, H.H.: Model-based performance risk analysis. Software Engineering, IEEE Transactions on 31, 3-20 (2005)

8. Wang, C.-C., Chen, C.-C.: Electronic commerce research in latest decade: A literature review. International Journal of Electronic Commerce Studies 1, 1-14 (2010)

9. Wang, P., Zhu, Z., Wang, Y.: A novel hybrid MCDM model combining the SAW, TOPSIS and GRA methods based on experimental design. Information Sciences 345, 27-45 (2016)

10. Saaty, T.L.: Decision making with the analytic hierarchy process. International journal of services sciences 1, 83-98 (2008)

11. Saaty, T.L.: Fundamentals of decision making and priority theory with the analytic hierarchy process. Rws Publications (2000)

12. Konidari, P., Mavrakis, D.: A multi-criteria evaluation method for climate change mitigation policy instruments. Energy Policy 35, 6235-6257 (2007) 
13. Behzadian, M., Otaghsara, S.K., Yazdani, M., Ignatius, J.: A state-of the-art survey of TOPSIS applications. Expert Systems with Applications 39, 13051$13069(2012)$

14. Hwang, C.-L., Yoon, K.: Multiple attribute decision making: methods and applications a state-of-the-art survey. Springer Science \& Business Media (2012)

15. Wu, F.-Y., Chuang, C.-C.: The optimal relationship between buyer and seller obtained using TOPSIS method. Journal of Advanced Management Science 1, 133-135 (2013) 